JOURNAL OF RESEARCH of the National Bureau of Standards - A. Physics and Chemistry

Vol. 74A, No. 4, July-August 1970

\title{
What is a Quasi-Particle?*1
}

\author{
J. R. Schrieffer \\ Department of Physics, University of Pennsylvania, Philadelphia, Pennsylvania
}

(October 10, 1969)

\begin{abstract}
The concept of a quasi-particle excitation in an interacting many-body system will be discussed from both the physical and the mathematical points of view. The physical origin of mass enhancement, wave function renormalization, interactions between quasi-particles, etc. will be presented. Landau's Fermi liquid theory, including the quasi-particle kinetic equation, will be reviewed. Finally, the domain of validity of the quasi-particle approximation will be discussed.
\end{abstract}

Key words: Density of states; Green's function; mass enhancement; quasi-particle; superconductors.

\section{Introduction}

Since the early work of Drude and of Sommerfeld [1], it has been clear that an independent-particle picture represents in a qualitatively correct manner the electronic properties of a metal. The electronic specific heat, the transport properties, the magnetic susceptibility, etc. are all roughly accounted for by elementary band theory, without recourse to explicit many-body effects. Exceptions to the rule are the plasma modes observed in the energy loss of fast charged particles, as well as cooperative phenomena such as superconductivity ferromagnetism, antiferromagnetism, etc.

The success of the independent-particle approximation (IPA) is particularly striking in view of the large ratio of interelectronic Coulomb energy to kinetic energy experienced by electrons in metals. A measure of this ratio is the electron density parameter $r_{s}$ defined essentially as the mean spacing between electrons, measured in units of the Bohr radius. For $r_{s} \ll 1$, the Coulomb interactions between electrons are weak compared to the kinetic energy effects, while if $r_{s} \gg 1$ the potential effects dominate the kinetic effects. For simple metals, $r_{s}$ is typically between two and six. Thus, one might expect qualitative changes in the properties

\footnotetext{
*An invited paper presented at the 3d Materials Research Symposium, Electronic Density of States, November 3-6, 1969, Gaithersburg, Md.

${ }^{1}$ This work was supported in part by the National Science Foundation.
}

of metals relative to the IPA since this approximation includes only the average Coulomb interaction between electrons. The "correlation energy" neglected by the IPA is of order 1 to $2 \mathrm{eV}$ per electron and is by no means trivial.

The qualitative reason that the IPA works so well is that typical measurements made on metals at normal temperatures $\left(T \ll T_{F} \sim 10^{5} \mathrm{~K}\right.$, where $T_{F}$ is the Fermi temperature) involve only the low lying excited states of the metal. There is good theoretical (and experimental) evidence that these many-body states are well characterized in terms of a set of elementary excitations, called quasi-particles, which for the interacting system play the same role as the excited electrons (above the Fermi surface) and the excited holes (below the Fermi surface) in the IPA. As for electrons and holes, these quasi-particles are labelled by a wave vector $\mathbf{k}$ and a spin orientation $s= \pm 1 / 2$. It is assumed that there is a sharp Fermi surface in the actual system as $T \rightarrow 0$, although its shape may depend on the manybody interactions. By convention, one measures the quasi-particle energies $\epsilon_{\mathbf{k}}$ relative to their (common) value on the Fermi surface so that $\epsilon_{\mathbf{k} F}=0$, where $\mathbf{k}_{F}$ is a wave vector on the Fermi surface.

At sufficiently low temperature, few quasi-particles are excited and therefore this dilute quasi-particle gas is nearly a "perfect" gas, in the sense that the quasiparticles rarely collide. Furthermore, at low temperature only low energy quasi-particles are excited. Since their intrinsic decay rate varies as $\epsilon_{\mathbf{k}}{ }^{2}$, they constitute long lived, weakly interacting excitations, thereby justi- 
fying their use as the building blocks for the low lying excitation spectrum.

There is no need in principle for the effective mass of the quasi-particles (q.p.) to be simply related to the free electron (or band structure) mass. For simple metals, it turns out that the q.p. mass $m^{*}$ is of the order of the free electron mass $m_{e}$, differing from it in most cases by a factor of less than two. The main source of the deviation of $m^{*}$ from $m_{e}$ (aside from band structure) is the electron-phonon interaction, which in general leads to an increase of $m^{*}$.

Unfortunately, there is at present no truly first principles proof of the above statements, i.e. the $1: 1$ correspondence of the low lying excited states of the noninteracting and interacting systems, a simple effective mass spectrum, long lifetimes of the quasi-particles, etc., although one has a proof that these statements are true to all orders in perturbation theory starting from the noninteracting states [2]. This lack of a rigorous foundation for the theory is not merely a mathematical nicety, since we know of many systems (e.g. the superconducting phase) which are not connected perturbatively with the noninteracting system; nevertheless, quasi-particles are still of use even in this case. One assumes that in normal systems (absence of cooperative effects) perturbation theory (or alternatively, adiabatic switching on of the interactions) gives the correct physics of the interacting system despite subtle nonanalytic effects which are likely present even in normal systems.

Thus far we have discussed only the excitation spectrum and not the many-body wave functions. Response functions (e.g. transport phenomena) require information about both quantities. The remarkable fact is that a suitably defined kinetic (or Boltzmann-like) equation for the quasi-particle distribution function gives an accurate account of the response of the system to long wavelength, low frequency perturbations such as electric and magnetic fields. This second property of quasiparticles is the heart of why the Drude-Sommerfeld scheme works well for nonequilibrium as well as equilibrium phenomena.

There is not time here to go into the details of the Landau theory of Fermi liquids, upon which the present theory of quasi-particles in metals is based. The excellent books of Pines and Nozieres [3] and of Nozieres [4] deal in depth with this topic. We would like, however, to give a brief sketch of the theory and to make a few comments about it.

\section{The Landau Picture of Fermi Liquids}

In the Landau picture one assumes that the low ener- gy excited states of the interacting system have energies well approximated by the form

$$
E\left(\delta n_{\mathbf{k} s}\right)=\sum_{\mathbf{k} s} \epsilon_{\mathbf{k} s} \delta n_{\mathbf{k} s}+\frac{1}{2} \sum_{\mathbf{k} s \mathbf{k}^{\prime} s^{\prime}} f_{\mathbf{k}}^{s \mathbf{k}^{\prime}} \delta n_{\mathbf{k} s} \delta n_{\mathbf{k}^{\prime} s^{\prime}}
$$

Here $\delta n_{\mathbf{k} s}$ is a measure of the quasi-particle (q.p.) occupation numbers. Assuming there is a well defined Fermi surface $S_{F}$ at zero temperature described by the wave vectors $\mathbf{k}_{F}$, one has

$$
\delta n_{\mathbf{k s}}=\left\{\begin{array}{c}
+1, \mathbf{k} s \text { outside } S_{F} \text { and occupied by a quasi- } \\
\text { electron } \\
-1, \mathbf{k} s \text { inside } S_{F} \text { and occupied by a quasi- } \\
\text { hole } \\
0, \text { otherwise. }
\end{array}\right.
$$

The zero-order q.p. energy $\epsilon_{\mathbf{k} s}$ is measured relative to the chemical potential $\mu$ so that $\epsilon_{\mathbf{k}_{F} s}=0$. One assumes that $\epsilon_{\mathbf{k} s}$ and its derivatives are continuous across $S_{F}$ and one makes the effective mass approximation

$$
\mathbf{v}_{\mathbf{k} s}=\frac{1}{\hbar} \nabla \epsilon_{\mathbf{k} s}=\frac{\hbar \mathbf{k}}{m^{*}}
$$

The approximation (2.2) often suffices, since one is usually interested in q.p. states $\mathbf{k} s$ in the immediate vicinity of $S_{F}$ (since $T \ll T_{F}$ ). The term involving $f_{\mathbf{k k}^{\prime}}^{s \mathbf{k}^{\prime}}$ represents the energy of interaction between quasi-particles. This function and $m^{*}$ are considered to be parameters to be determined from experiment or to be roughly estimated from a more fundamental theory.

Landau argued that if one views the quasi-particles as being described by wave packets whose extent is large compared to the wavelength of a q.p. at the Fermi surface $\left(\lambda_{F} \sim 2 \pi / k_{F} \sim 10^{-8} \mathrm{~cm}\right.$ in metals) then one can define a distribution function $\delta n_{\mathbf{k s}}(\mathbf{r}, t)$ for q.p.'s which plays the role of the single particle distribution function $f(\mathbf{r}, \mathbf{p}, t)$ in kinetic theory. This concept is reasonable as long as $\delta n_{\mathbf{k} s}(\mathbf{r}, t)$ varies slowly in space (compared to $\lambda_{F}$ ) and in time (compared to $\hbar / \mu$ ). By the usual arguments of kinetic theory one can write down a kinetic (LandauBoltzmann) equation for $\delta n$.

$$
\begin{array}{r}
\frac{\partial \delta n_{\mathbf{k s} s}(\mathbf{r}, t)}{\partial t}+\mathbf{v}_{\mathbf{k}} \cdot \nabla_{\mathbf{r}} \delta \bar{n}_{\mathbf{k} s}(\mathbf{r}, t)-\mathscr{F}_{\mathbf{k}} \cdot \mathbf{v}_{\mathbf{k}} \delta\left(\boldsymbol{\epsilon}_{\mathbf{k} s}\right) \\
=I\left(\delta n_{\mathbf{k} s}\right) .
\end{array}
$$

$\mathscr{F}_{\mathbf{k}}$ and $I\left(\delta n_{\mathbf{k} s}\right)$ are the external force acting on the q.p. and the collision integral respectively, while $\nabla_{\mathbf{r}} \delta \bar{n}_{\mathbf{k} s}$ is defined by

$$
\begin{aligned}
\nabla_{\mathbf{r}} \delta \bar{n}_{\mathbf{k} s}(\mathbf{r}, t)= & \nabla_{\mathbf{r}} \delta n_{\mathbf{k} s}(\mathbf{r}, t) \\
& +\delta\left(\epsilon_{\mathbf{k s} s}\right) \sum_{\mathbf{k}^{\prime} s^{\prime}} f_{\mathbf{k k}^{\prime}}^{s s^{\prime}} \nabla_{\mathbf{r}^{\prime}} \delta n_{\mathbf{k}^{\prime} s^{\prime}}(\mathbf{r}, t) .
\end{aligned}
$$


The term $\nabla_{1} \delta n_{\mathbf{k} s}$ in (2.4) describes the conventional streaming flow of q.p.'s familiar from kinetic theory. The other term, arising from the interactions, may be viewed as a dragging along of the ground state particles by the inhomogeneous distribution of quasi-particles, each q.p. dragging along its own cloud.

Naively, one might guess that the particle current density $\mathbf{J}(\mathbf{r}, t)$ at point $\mathbf{r}$ may be expressed as $\sum_{\mathbf{k} s} \mathbf{v}_{\mathbf{k} s} \delta n_{\mathbf{k} s}(\mathbf{r}, t)$. This is not true, but rather

$$
\begin{aligned}
& \mathbf{J}(\mathbf{r}, t)=\sum_{\mathbf{k} s} \mathbf{v}_{\mathbf{k} s} \delta \bar{n}_{\mathbf{k} s}(\mathbf{r}, t) \\
& \quad=\sum_{\mathbf{k} s}\left[\mathbf{v}_{\mathbf{k} s}+\sum_{\mathbf{k}^{\prime} s^{\prime}} f_{\mathbf{k} \mathbf{k}^{\prime}}^{s s^{\prime}} \mathbf{v}_{\mathbf{k}^{\prime} s^{\prime}} \delta\left(\boldsymbol{\epsilon}_{\mathbf{k}^{\prime} s^{\prime}}\right)\right] \delta n_{\mathbf{k} s}(\mathbf{r}, t) .
\end{aligned}
$$

The $\mathbf{v} \delta n$ term represents the current of the quasi-particle, while the term involving $f$ represents the current of the ground state particles being "dragged along" with the q.p. It is clear from the kinetic equation (2.3) that this definition is correct since the continuity equation

$$
\frac{\partial \rho}{\partial t}+\nabla \cdot \mathbf{J}=0
$$

is satisfied by $\mathbf{J}$ if we use the fact that

$$
\rho=\rho_{0}+\sum_{\mathbf{k} s} \delta n_{\mathbf{k s}}(\mathbf{r}, t) .
$$

Roughly speaking $f_{\mathbf{k k}^{\prime}}^{s \prime^{\prime}}$ behaves like a velocity dependent potential acting on particles in $\mathbf{k}$ and $\mathbf{k}^{\prime}$. A change in $\delta n_{\mathbf{k} s}$ acts on the particle in $\mathbf{k}^{\prime} s^{\prime}$ like a vector potential would, and induces a current even though the $\mathbf{k}^{\prime}$ wavefunction does not change, like the Meissner effect in a superconductor.

\section{Quasi-Particles in Metals}

The above picture is suitable for a system like $\mathrm{He}^{3}$ which is translationally invariant in its ground state and has only the Fermion degrees of freedom. Metals are clearly different: they are invariant only under the translation group of the crystal lattice and have lattice vibrations as well as electronic degrees of freedom. How much of the Landau picture survives? The "noninteracting system" is presumably now the IPA in which the Coulomb interactions between electrons are treated in the mean field approximation. In this case the one particle states are labelled by a wave vector $\mathbf{k}$ (restricted to the first Brillouin zone), a band index $n$ and the spin $s$. We lump $n$ and $\mathbf{k}$ together for now. There is a sharp Fermi surface at $T=0$ and excited states are given by the usual electron and hole excitations. Since the Coulomb interaction has full transla- tion invariance, $\mathbf{k}$ remains a good quantum number to describe the quasi-particles, although the ground state of the actual system may be the transform of some excited state of the IPA system, due to changes in shape of the Fermi surface. Luttinger and Nozieres [2] have shown to all orders in perturbation theory that the volume of $\mathbf{k}$ space inside $S_{F}$ remains fixed, as it must for the Landau picture to make sense. The energy expression (2.1) still holds but $f_{\mathbf{k k}}^{s \mathbf{k}^{\prime}}$ is in general a function having only the symmetry of the crystal, rather than full the rotational symmetry present for say $\mathrm{He}^{3}$. The effective mass expression for $\mathbf{v}_{\mathbf{k}}$ still holds except $1 / m^{*}$ is in general a second rank tensor having only the symmetry of the crystal. If the crystalline anisotropy of $m^{*}$ and $f$ are very weak (as for $\mathrm{Na}$ ) then the identity

$$
\frac{m^{*}}{m}=\frac{1+F_{1}^{s}}{3}
$$

relating the effective mass ratio and the spin symmetric $l=1 \operatorname{term} F_{1}{ }^{s}$ in a Legendre polynomial expansion of $f_{\mathbf{k k}^{\prime}}^{s s^{\prime}}$ for a true Fermi liquid is valid. Here, if $N(0)$ is the density of single particle states of one spin orientation at the Fermi surface, then

$$
F_{1}^{s}=N(0)\left[f_{1}^{\uparrow \uparrow}+f_{1}^{\uparrow}\right]
$$

and

$$
f_{\mathbf{k} \mathbf{k}^{\prime}}^{s s^{\prime}}=\sum_{l=0}^{\infty} f_{l}^{s s^{\prime}} P_{l}\left(\cos \hat{\mathbf{k}} \mathbf{k}^{\prime}\right)
$$

Thus, for nearly free electron metals the low temperature electronic specific heat, i.e. $m^{*} / m$, determines $F_{1}$. Other pieces of information about $f_{\mathbf{k} \mathbf{k}^{\prime}}^{s s^{\prime}}$ can be extracted from other experiments such as the anomalous skin effect, Azbel-Kaner cyclotron resonance, de Haas van Alphen effect, dynamic magnetic susceptibility, etc. Presumably the $f_{l}$ drop off rapidly with increasing $l$ so only $l=0,1$ and perhaps 2 need be retained. For nonfree electron metals, it appears that the anisotropy of $f$ is so large that unravelling this function will be quite involved. However, we know that the transport and the $d H v A$ measurements have already given us a great deal of information about $1 / m^{*}$ in complex metals. When combined with band structure calculations these measurements give information on the many-body effects in these systems. As we mentioned earlier, most of the $\mathrm{m}^{*} / \mathrm{m}$ effect is due to the phonons, for which a reasonably good first principles calculation is becoming possible, in many metals. A careful comparison here would provide an important check on the approximation of band theory and of the approximate methods presently used in many-body theory. 
Another problem is that the phonons complicate the kinetic equation and the current density expression, since the phonons carry momentum and energy. The necessary generalizations of the Landau theory have been worked by Prange and Kadanoff [5], althoügh we do not have time to discuss these questions here.

\section{Green's Function Picture of Quasi-Particle}

An alternative way of viewing quasi-particles, which is more general than the Landau theory, is through the Green's function scheme of many-body theory $[3,4,6,7]$. Suppose that an interacting system of $N$ electrons is initially prepared to be in its exact ground state, $|0, N\rangle$. If $c_{\mathbf{k} s}^{+}$creates a (bare) Bloch electron in state $\mathbf{k} s$, then we desire the probability distribution $P_{\mathbf{k} s}(E)$ of the energy for the $N+1$ particle state $\Phi_{\mathbf{k} s}^{N+1}+\mathbf{l}$ defined by

$$
\left|\Phi_{\mathbf{k} s}^{N+1}\right\rangle=c_{\mathbf{k} s}^{+}|0, N\rangle .
$$

In general, $\Phi$ is not an eigenstate of the full Hamiltonian so that $\Phi$ does not have a sharply defined energy, i.e. $P_{\mathbf{k} s}(E)$ is not a delta function. The rules of quantum mechanics tell us that if the states $|n, N+1\rangle$ are the exact energy eigenstates of the $N+1$ particle problem then

$$
\begin{array}{r}
P_{\mathbf{k} s}(E)=\sum_{n}\left|\left\langle n, N+1\left|c_{\mathbf{k} s}^{+}\right| 0, N\right\rangle\right|{ }^{2} \delta(E \\
\left.-\left[E_{n}^{N+1}-E_{0}^{N}\right]+\mu\right) \\
=\sum_{n}\left|\left\langle n, N+1 \mid \Phi_{\mathbf{k} s}^{N+1}\right\rangle\right|{ }^{2} \delta(E \\
\left.-\left[E_{n}^{N+1}-E_{0}^{N}\right]+\mu\right),
\end{array}
$$

where the $E_{n}^{N+1}$ are the energy eigenvalues of the many-body system. Within the IPA, $P_{\mathbf{k s}}(E)$ is a delta function, since $c_{\mathbf{k} s}^{+}$creates a Bloch state electron, which by definition is an exact single particle eigenstate of energy $\epsilon_{\mathbf{k} s}$. Thus, for the independent particle approximation (IPA)

$$
P_{\mathbf{k} s}^{\mathrm{IPA}}(E)=\left\{\begin{array}{cc}
\delta\left(E-\epsilon_{\mathbf{k} s}\right) & \epsilon_{\mathbf{k} s}>0 \\
0 & \epsilon_{\mathbf{k} s}<0 .
\end{array}\right.
$$

Clearly, according to the Pauli Principle $P$ is zero if one tries to add a particle to an already filled state, $\epsilon_{\mathrm{ks}}<0$. For the interacting system, $P$ will be a complicated (positive) function of $E$ in general, whose shape depends on the value of $\mathbf{k} s$. The essential point is that if $\mathbf{k}$ is slightly above the Fermi surface, $P_{\mathbf{k s}}(E)$ will consist of a narrow high peak centered about a "quasi-particle energy,"

$$
E=\tilde{\epsilon}_{\mathbf{k} s}, \frac{1}{\hbar} \nabla_{\mathbf{k}} \tilde{\epsilon}_{\mathbf{k} s} \cong \frac{\hbar \mathbf{k}}{m^{*}},
$$

plus a background continuum which in general has a rather smooth behavior, as sketched in figure $\mathbf{l}$ for $\mathbf{k}$ just above the Fermi surface. The half-width of the peak $\Gamma_{\mathbf{k} s}$, gives the intrinsic decay rate of the quasi-particle according to $1 / \tau_{\mathbf{k} s}=2 \Gamma_{\mathbf{k} s} / \hbar$. Perturbation theoretic arguments show that $\Gamma$ goes to zero as $\epsilon_{\mathrm{ks} s}{ }^{2}$ for $\epsilon_{\mathrm{ks} s} \ll \mu$ so that the fractional width (or the reciprocal " $Q$ " of the particle) varies as $\tilde{\boldsymbol{\epsilon}}_{\mathbf{k} s}$, showing the quasi-particle to be a well defined excitation near the Fermi surface. To complete the story, one considers hole states defined by

$$
\left|\bar{\Phi}_{\mathbf{k} s}^{N-1}\right\rangle=C_{\mathbf{k} s}|0, N\rangle,
$$

Like $\Phi_{\mathbf{k} s}^{N+1}, \Phi_{\mathbf{k} s}^{N-1}$ is not an eigenstate of energy for the interacting system. Thus, the probability distribution of $E$ for the hole state, which is defined by

$$
\begin{aligned}
& \bar{P}_{\mathbf{k} s}(E)= \sum_{n}\left|\left\langle n, N-1 \mid \bar{\Phi}_{\mathbf{k} s}^{N-1}\right\rangle\right|^{2} \\
& \delta\left(E+\left[E_{n}^{N-1}-E_{0}^{N}\right]+\mu\right) \\
&=\sum_{n} \mid\left\langle n, N-1\left|c_{\mathbf{k} s}\right|\right.0, N\rangle\left.\right|^{2} \\
& \delta\left(E+\left[E_{n}^{N-1}-E_{0}^{N}\right]+\mu\right)
\end{aligned}
$$

is not a delta function in general. Note the change of sign of the excitation energy term in the delta functions appearing in (4.2) and (4.6). This ensures that at zero temperature the holes have negative energy and electrons have positive energy. Within the IPA, $\bar{\Phi}_{\mathbf{k} s}^{N-1}$ is an eigenstate of $H$ and $\bar{P}_{\mathbf{k} s}^{I P A}(E)$ given by

$$
\bar{P}_{\mathbf{k} s}^{\mathrm{IPA}}(E)= \begin{cases}0 & \epsilon_{k s}>0 \\ \delta\left(E-\epsilon_{\mathbf{k} s}\right) & \epsilon_{\mathbf{k} s}<0 .\end{cases}
$$

For the interacting system, if $\mathbf{k} s$ is just below the Fermi surface, a narrow, high "quasi-hole" peak centered about $\tilde{\epsilon}_{\mathbf{k} s}$ appears in $\bar{P}_{\mathbf{k} s}(E)$, with a continuum background again occurring as sketched in figure 2 . The quasi-hole and quasi-electron energies presumably

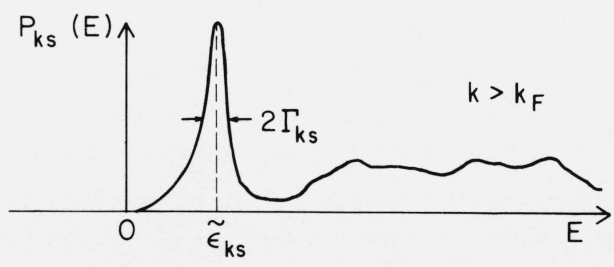

FigURE 1. Probability distribution $\mathrm{P}_{k s}(\mathrm{E})$ for a "quasi-particle" corresponding to a bare Bloch state $\mathrm{ks}$ for $\mathrm{k}>\mathrm{k}_{F}$. 


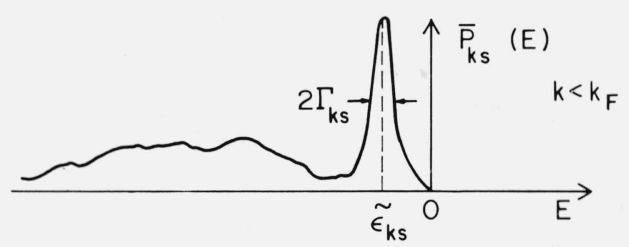

FIgURE 2. Probability distribution $\mathrm{P}_{k s}(\mathrm{E})$ for a "quasi-hole" state with $\mathrm{k}<\mathrm{k}_{F}$.

join on smoothly at the Fermi surface so that $m^{*}$ is continuous across $S_{F}$.

At nonzero temperature, one makes a statistically weighted average over initial states, rather than considering only the ground state $|0, N\rangle$. In this case the electron and hole probability distributions overlap, in that they are both nonzero for $E>0$ and for $E<0$. The overlapping corresponds to creating or destroying thermally excited quasi-particles, amongst other things. From the Fermi statistics of electrons, there follows the rigorous sum rule

$$
\int_{-\infty}^{\infty}\left[P_{\mathbf{k s}}(E)+\bar{P}_{\mathbf{k s}}(E)\right] d E=1 .
$$

One advantage of the Green's function description is that it allows the concept of quasi-particles to be usefully extended to systems which are not related by an adiabatic transform or by perturbation theory to the noninteracting states. For example, in a superconductor, $P_{\mathbf{k s}}(E)$ shows at low temperature a sharp peak at the "quasi-particle" energy

$$
E=E_{\mathbf{k} s} \equiv \sqrt{\epsilon_{\mathbf{k} s}^{2}+\Delta_{\mathbf{k}}^{2}},
$$

while $\bar{P}_{\mathrm{ks}}(E)$ shows a sharp peak at $E=-E_{\mathrm{k} s}$ as sketched in figure 3 . In addition, $P$ and $\bar{P}$ show background continua like in the normal metal. Note however that as $\mathbf{k}$ approaches the Fermi surface there is an energy gap between the quasi-hole and quasi-electron peaks, the gap being $2 \Delta_{\mathbf{k}_{F}}$, as is well known from the pairing theory. Thus a minimum energy $2 \Delta_{\mathbf{k}_{r}}$ is required to make a single-particle excitation in a superconductor (i.e. creation of a quasi electron-hole pair).

We should mention that the "background continuum" mentioned above corresponds physically to the creation of more complicated excitations, such as a quasi-particle plus electron-hole pairs, phonons, plasmons, etc. Generally these extra excitations are not strongly coupled together and therefore an incoherent (smooth) continuum appears. In special cases, how-
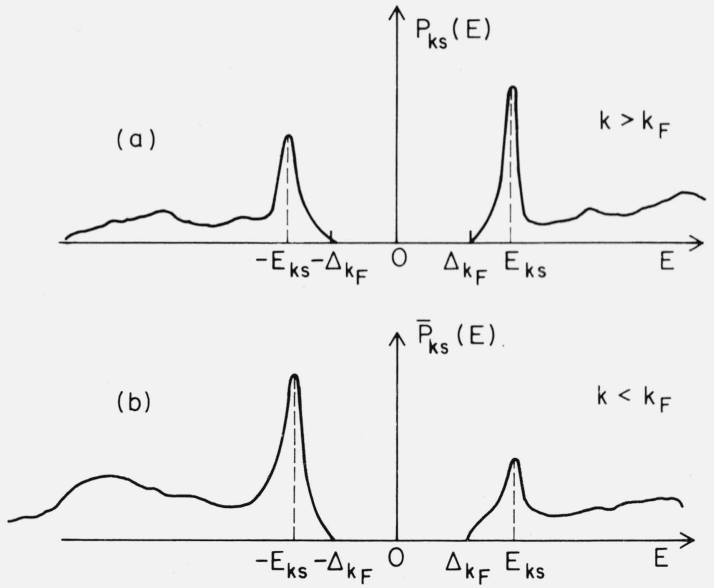

FigURE 3. $\quad \mathrm{P}_{k s}(\mathrm{E})$ and $\overline{\mathrm{P}}_{k s}(\mathrm{E})$ for a superconductor showing $($ a) sharp peak at $\mathrm{E}=\mathrm{E}_{k s}$ in $\mathrm{P}_{k s}(\mathrm{E})$ and $\left(\right.$ b) peak in $\overline{\mathrm{P}}_{k s}(\mathrm{E})$ at $\mathrm{E}=-\mathrm{E}_{k s}$.

ever, resonant scatterings states of the excitations can appear, an example being the quasi-bound state of a hole and a plasmon, as Hedin et al. [8] have discussed.

There is a great deal more one should say about quasi-particles. The interested reader can follow the story further in the books mentioned above and the references contained therein. It is the present author's view that a clearer physical picture of such questions as "drag currents," "back flow," "screening," quasiparticle interactions (both forward and nonforward scattering amplitudes), particularly in real metals, deserve careful attention in the future.

\section{References}

[1] See C. Kittel, Introduction to Solid State Physics, Chapter 10, J. Wiley \& Sons, Inc., New York, and J. Ziman, Principles of the Theory of Solids, Cambridge U. Press (1964).

[2] Luttinger, J. M., and Nozieres, P., Phys. Rev. 127, 1423, 1431 (1962).

[3] Pines, D., and Nozieres, P., The Theory of Quantum Liquids, Vol. I, W. A. Benjamin, Inc., New York (1966).

[4] Nozieres, P., Interacting Fermi Systems, W. A. Benjamin, Inc., New York (1964).

[5] Prange, R. E., and Kadanoff, L. P., Phys. Rev. 134, A566 (1964).

[6] Abrikosov, A. A., Gorkov, L. P., and Dzysloshinskii, I. E., Methods of Quantum Field Theory in Statistical Physics, Prentice-Hall, Inc. (1963).

[7] Schrieffer, J. R., Theory of Superconductivity, W. A. Benjamin, Inc., New York (1964).

[8] Hedin, L., Lundqvist, B. I., and Lundqvist, S., this Symposium.

(Paper 74.A4-620) 